Pure and Applied Mathematics Quarterly

Volume 5, Number 4

(Special Issue: In honor of

John Tate, Part 1 of 2)

$1219-1252,2009$

\title{
Motivic Complexes over Finite Fields and the Ring of Correspondences at the Generic Point
}

\author{
James S. Milne and Niranjan Ramachandran
}

To John Tate in admiration and gratitude.

\begin{abstract}
Already in the 1960s Grothendieck understood that one could obtain an almost entirely satisfactory theory of motives over a finite field when one assumes the full Tate conjecture. In this note we prove a similar result for motivic complexes. In particular Beilinson's $\mathbb{Q}$-algebra of "correspondences at the generic point" is then defined for all connected varieties. We compute it for all smooth projective varieties (hence also for varieties birational to such a variety).
\end{abstract}

Keywords: correspondence, motive, Tate conjecture.

\section{Contents}




\section{Introduction}

More than forty years after Grothendieck predicted that the standard cohomology functors factor through a tannakian category of pure motives, we still do not know how to construct such a category. However, when the field is finite and one assumes the full Tate conjecture, there is an almost entirely satisfactory theory of pure motives. According to Deligne (1994, 1.4), this was known to Grothendieck, but it was re-discovered by Langlands and Rapoport (1987), who used it to state a conjecture, more precise than earlier attempts by Langlands, on the structure of the points modulo a prime on a Shimura variety. For a detailed description of the category, see Milne 1994.

It is generally hoped that the standard cohomology functors to triangulated categories will factor through a triangulated category of motivic complexes with $t$-structure whose heart is (defined to be) the category of mixed motives (see, for example, Deligne 1994, §3). We show that, over a finite field, a triangulated category of motivic complexes exists with the expected properties if and only if the Tate conjecture holds and homological equivalence coincides with rational equivalence with $\mathbb{Q}$-coefficients (see Theorems 4.2 and 5.3 for more precise statements). Moreover, then a category of effective motivic complexes exists with the properties (A,B,C) of Beilinson 2002, and so there is a well-defined semisimple $\mathbb{Q}$ algebra of "correspondences at the generic point" attached to every variety over a finite field. We compute this $\mathbb{Q}$-algebra for smooth projective varieties (hence also for varieties birational to such a variety). As this requires the generalized Tate conjecture (in the sense of Grothendieck 1968, §10), we begin by giving an elementary proof that this follows from the usual Tate conjecture. 


\section{Notations}

A variety is a geometrically-reduced separated scheme of finite type over a field. For a variety $X$ over a perfect field $k$ of characteristic $p \neq 0$ and algebraic closure $\bar{k}$, we set

$$
\begin{aligned}
& H_{l}^{i}(X)=H_{\mathrm{et}}^{i}\left(X_{\bar{k}}, \mathbb{Q}_{l}\right), \quad \text { if } l \neq p, \text { and } \\
& H_{p}^{i}(X)=H_{\mathrm{crys}}^{i}(X / W) \otimes \mathbb{Q}, \quad W=W(k) .
\end{aligned}
$$

We use $(r)$ to denote a Tate twist, and we write hom $(l)$ for the equivalence relation on the space $Z^{*}(X)$ of algebraic cycles defined by $H_{l}$. Similarly, we write num and rat for numerical and rational equivalence. For an adequate equivalence relation $\sim, Z_{\sim}^{i}(X)=Z^{i}(X) / \sim$ and $Z_{\sim}^{i}(X)_{\mathbb{Q}}=Z_{\sim}^{i}(X) \otimes \mathbb{Q}$. For example, $Z_{\text {rat }}^{i}(X)$ is the Chow group $C H^{i}(X)$.

By a functor between additive categories, we mean an additive functor. A functor $F: \mathcal{C} \rightarrow \mathcal{C}^{\prime}$ of triangulated categories together with an isomorphism of functors $F \circ T \approx T^{\prime} \circ F$ is said to be triangulated (formerly, exact; Verdier 1977, p4) if it takes distinguished triangles to distinguished triangles.

A triangulated category with $t$-structure (Gelfand and Manin 1996, IV 4.2, p278) will be referred to simply as a $t$-category. All $t$-structures will be assumed to be bounded (i.e., $\bigcup_{n \geq 0} \mathcal{D}^{\leq n}=\mathcal{D}=\bigcup_{n \geq 0} \mathcal{D}^{\geq-n}$ ) and nondegenerate (i.e., $\left.\bigcap_{n \geq 0} \mathcal{D}^{\leq-n}=0=\bigcap_{n \geq 0} \mathcal{D}^{\geq n}\right)$.

The symbol $\mathbb{F}$ denotes an algebraic closure of $\mathbb{F}_{p}$, and the algebraic closure of $\mathbb{Q}$ in $\mathbb{C}$ is denoted $\mathbb{Q}^{\text {al }}$. Reductive groups are not required to be connected. Isomorphisms are denoted $\approx$ and canonical isomorphisms $\simeq$.

\section{The generalized Tate conjecture}

In this section, $k$ is the subfield $\mathbb{F}_{q}$ of $\mathbb{F}$, and $l \neq p$.

1.1 By the full Tate conjecture for a smooth complete variety $X$ over $k$ and an $r \geq 0$, we mean the statement that the order of the pole of the zeta function $Z(X, t)$ at $t=q^{-r}$ is equal to the rank of the group of numerical equivalence 
classes of algebraic cycles of codimension $r$ on $X$. If the full Tate conjecture holds for $X$ and $r$, then, for all $l \neq p$,

$T^{r}(X, l)$ : the cycle class map $Z^{r}(X) \otimes \mathbb{Q}_{l} \rightarrow H_{l}^{2 r}(X)(r)^{\mathrm{Gal}(\mathbb{F} / k)}$ is surjective, and $E^{r}(X, l)$ : the quotient map $Z_{\operatorname{hom}(l)}^{r}(X)_{\mathbb{Q}} \rightarrow Z_{\text {num }}^{r}(X)_{\mathbb{Q}}$ is injective (i.e., $\operatorname{hom}(l)$ and num coincide with $\mathbb{Q}$-coefficients).

Conversely, if $T^{r}(X, l)$ and $E^{r}(X, l)$ hold for a single $l$, then the full Tate conjecture holds for $X$ and $r$ (Tate 1994, $\S 2$ ). The statement $T^{r}(X, l)$ is the Tate conjecture for $X, r$, and $l .^{1}$

\section{Statement of the generalized Tate conjecture}

Define a Tate structure to be a finite-dimensional $\mathbb{Q}_{l}$-vector space with a linear (Frobenius) map $\varpi$ whose characteristic polynomial lies in $\mathbb{Q}[T]$ and whose eigenvalues are Weil $q$-numbers, i.e., algebraic numbers $\alpha$ such that, for some integer $m$ (called the weight of $\alpha$ ), $|\rho(\alpha)|=q^{m / 2}$ for every homomorphism $\rho: \mathbb{Q}[\alpha] \rightarrow \mathbb{C}$, and, for some integer $n, q^{n} \alpha$ is an algebraic integer. When the eigenvalues are all of weight $m$ (resp. algebraic integers, resp. semisimple), we say that $V$ is of weight $m$ (resp. effective, resp. semisimple). For example, for any smooth complete variety $X$ over $k, H_{l}^{i}(X)$ is an effective Tate structure of weight $i / 2$ (Deligne 1980), which is semisimple if $X$ is an abelian variety (Weil 1948b, no. 70) or if the full Tate conjecture holds for $X \times X$ (Milne 1986b, 8.6).

Let $X$ be a smooth complete variety over $k$. For each $r$, let $F_{a}^{r} H_{l}^{i}(X) \subset H_{l}^{i}(X)$ denote the subspace of classes with support in codimension at least $r$, i.e.,

$$
F_{a}^{r} H_{l}^{i}(X)=\bigcup_{U} \operatorname{Ker}\left(H_{l}^{i}(X) \rightarrow H_{l}^{i}(U)\right)
$$

where $U$ runs over the open subvarieties of $X$ such that $X \backslash U$ has codimension $\geq r$.

EXAMPLE 1.2 If $Z$ is a smooth closed subvariety of $X$ of codimension $r$, then there is an exact Gysin sequence

$$
\cdots \rightarrow H_{l}^{i-2 r}(Z)(-r) \rightarrow H_{l}^{i}(X) \rightarrow H_{l}^{i}(U) \rightarrow \cdots, \quad U=X \backslash Z,
$$

\footnotetext{
${ }^{1}$ More precisely, it is Conjecture 1 of Tate 1965. Statement $E^{r}(X, l)$ is a variant of the "conjectural statement" ( $\left.\mathbf{a}^{\prime}\right)$ of Tate 1965. Our notation follows that of Tate 1994.
} 
(e.g., Milne 1980, VI 5.4), and so the kernel of $H_{l}^{i}(X) \rightarrow H_{l}^{i}(U)$ is an effective Tate structure of weight $i$ whose twist by $\mathbb{Q}_{l}(r)$ is still effective.

Conjecture 1.3 (Generalized Tate conjecture; cf. Grothendieck 1968, 10.3.). For a smooth complete variety $X$ over $k$, every semisimple Tate substructure $V \subset H_{l}^{i}(X)$ such that $V(r)$ is still effective is contained in $F_{a}^{r} H_{l}^{i}(X)$.

REMARK 1.4 Let $X$ be a smooth complete variety over $k$. For any $i$ and $r$, the set of eigenvalues $\alpha$ of $\varpi_{X}$ on $H_{l}^{i}(X)$ such that $\alpha / q^{r}$ is an algebraic integer is stable under Galois conjugation. Therefore, there is a subspace $F_{b}^{r} H_{l}^{i}(X)$ of $H_{l}^{i}(X)$ that becomes the sum of the eigenspaces of these $\alpha$ over $\mathbb{Q}_{l}^{\text {al }}$. It is the largest semisimple Tate substructure of $H_{l}^{i}(X)$ whose twist by $\mathbb{Q}_{l}(r)$ is still effective, and so the generalized Tate conjecture 1.3 is the statement:

$$
F_{b}^{r} H_{l}^{i}(X) \subset F_{a}^{r} H_{l}^{i}(X) .
$$

EXAmple 1.5 Let $Z^{\prime}$ be a closed irreducible subvariety of $X_{\mathbb{F}}$ of codimension $r$. Then

$$
H_{Z^{\prime}}^{2 r}\left(X_{\mathbb{F}}, \mathbb{Q}_{l}(r)\right) \rightarrow H^{2 r}\left(X_{\mathbb{F}}, \mathbb{Q}_{l}(r)\right) \rightarrow H^{2 r}\left(X_{\mathbb{F}} \backslash Z^{\prime}, \mathbb{Q}_{l}(r)\right)
$$

is exact, and $H_{Z^{\prime}}^{2 r}\left(X_{\mathbb{F}}, \mathbb{Q}_{l}(r)\right) \simeq \mathbb{Q}_{l}$; moreover, the image of 1 under the first map is the cohomology class of $Z^{\prime}$ (cf. Milne 1980, p269). For any open $U \subset X$, the kernel of

$$
H_{l}^{2 r}(X)(r) \rightarrow H_{l}^{2 r}(U)(r)
$$

is spanned by the cohomology classes of the irreducible components of $(X \backslash U)_{\mathbb{F}}$, and some power of $\varpi_{X}$ acts as 1 on it. On the other hand, $F_{b}^{r} H_{l}^{2 r}(X)(r)$ is the largest subspace of $H_{l}^{2 r}(X)(r)$ on which some power of $\varpi$ acts as 1 . Thus, the generalized Tate conjecture with $i=2 r$ states that this subspace is spanned by the classes of algebraic cycles of codimension $r$ on $X_{\mathbb{F}}$. This is the Tate conjecture stated over $\mathbb{F}$ rather than $\mathbb{F}_{q}$.

\section{The Tate conjecture implies the generalized Tate conjecture}

Recall that, for a proper map $\pi: Y \rightarrow X$ of smooth varieties over an algebraically closed field, the Gysin map

$$
\pi_{*}: H^{i}\left(Y, \mathbb{Q}_{l}\right) \rightarrow H^{i-2 c}\left(X, \mathbb{Q}_{l}(-c)\right), \quad c=\operatorname{dim} Y-\operatorname{dim} X,
$$


is defined to be the Poincaré dual of

$$
\pi^{*}: H_{c}^{2 d-i}\left(X, \mathbb{Q}_{l}(d)\right) \rightarrow H_{c}^{2 d-i}\left(Y, \mathbb{Q}_{l}(d)\right), \quad d=\operatorname{dim} Y
$$

(Milne 1980, VI 11.6). We shall need to know that these maps are compatible with restriction to open subvarieties.

Lemma 1.6 Let $\pi: Y \rightarrow X$ be a proper map of smooth complete varieties over an algebraically closed field, and let $j: U \hookrightarrow X$ an open immersion. Then the commutative diagram at left gives rise to the commutative diagram at right:

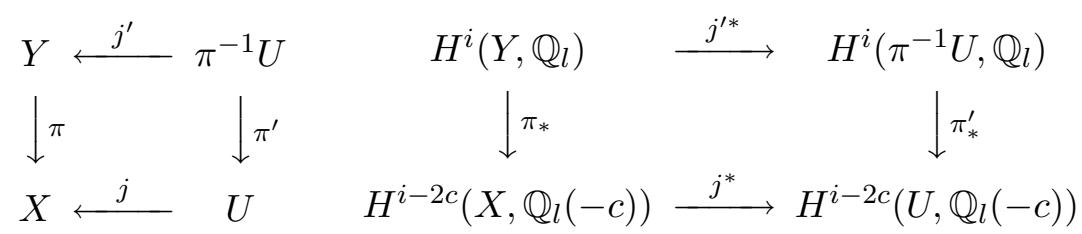

Proof. Exercise for the reader.

Proposition 1.7 Every effective semisimple Tate structure is isomorphic to a Tate substructure of $H_{l}^{*}(A)$ for some abelian variety $A$ over $\mathbb{F}_{q}$.

Proof. We may assume that the Tate structure $V$ is simple. Then $V$ has weight $m$ for some $m \geq 0$, and the characteristic polynomial $P(T)$ of $\varpi$ is a monic irreducible polynomial with coefficients in $\mathbb{Z}$ whose roots all have real absolute value $q^{m / 2}$. According to Honda's theorem (Honda 1968; Tate 1968), $P(T)$ is the characteristic polynomial of an abelian variety $A$ over $\mathbb{F}_{q^{m}}$. Let $B$ be the abelian variety over $\mathbb{F}_{q}$ obtained from $A$ by restriction of the base field. The eigenvalues of the Frobenius map on $H_{l}^{1}(B)$ are the $m^{\text {th }}$-roots of the eigenvalues of the Frobenius map on $H_{l}^{1}(A)$, and it follows that $V$ is a Tate substructure of $H_{l}^{m}(B)$.

LEMMA 1.8 Let $z$ be an algebraic cycle of codimension $\operatorname{dim} T+r$ on the product $T \times X$ of two smooth complete varieties over $k$ (i.e., $z$ is an algebraic correspondence of degree $r$ from $T$ to $X$ ). Assume that the push-forward of $z$ on $X$ is nonzero. Then the image of the map

$$
z_{*}: H_{l}^{i-2 r}(T)(-r) \rightarrow H_{l}^{i}(X)
$$

defined by $z$ is contained in $F_{a}^{r} H_{l}^{i}(X)$. 
Proof. Let $p, q$ denote the projection maps $T \times X \rightrightarrows T, X$, and let $[z]$ denote the cohomology class of $z$ in $H_{l}^{2 d_{T}+2 r}(T \times X)\left(d_{T}+r\right), d_{T}=\operatorname{dim} T$. Then

$$
z_{*}(a) \stackrel{\text { def }}{=} q_{*}\left([z] \cup p^{*}(a)\right), \quad a \in H_{l}^{i-2 r}(T)(-r) .
$$

As the push-forward $q_{*}(z)$ of $z$ is nonzero, its support $Z$ has codimension $r{ }^{2}$ Let $U=X \backslash Z$. Then $z$ has support in $T \times Z$, and so $[z]$ maps to zero in $H_{l}^{2 d_{T}+2 r}(T \times U)\left(d_{T}+r\right)$. According to (1.6), the diagram

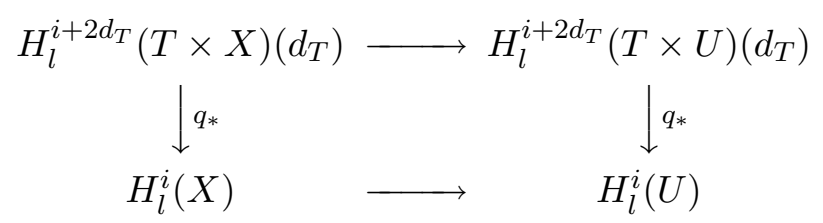

commutes, which shows that $z_{*}(a)$ maps to zero in $H_{l}^{i}(U)$, and therefore lies in $F_{a}^{r} H_{l}^{i}(X)$.

Lemma 1.9 Let $X$ be a smooth complete variety over $k$ and let $i, r \in \mathbb{N}$. If there exists a smooth complete variety $T$ such that

- $H_{l}^{i-2 r}(T)$ is a semisimple Tate structure,

- the Tate conjecture $T^{\operatorname{dim}(T)+r}(T \times X, l)$ holds, and

- $F_{b}^{r} H_{l}^{i}(X)(r)$ is isomorphic to a Tate substructure of $H_{l}^{i-2 r}(T)$

then $F_{b}^{r} H_{l}^{i}(X) \subset F_{a}^{r} H_{l}^{i}(X)$.

Proof. Let $d=\operatorname{dim}(T)$ and let $V$ be a Tate substructure of $H_{l}^{i-2 r}(T)$ for which there exists an isomorphism $f: V(-r) \rightarrow F_{b}^{r} H_{l}^{i}(X)$. Then

$$
\begin{aligned}
H_{l}^{2 d+2 r}(T \times X)(d+r) & \supset H_{l}^{2 d+2 r-i}(T)(d+r) \otimes H_{l}^{i}(X) \\
& \simeq \operatorname{Hom}\left(H_{l}^{i-2 r}(T)(-r), H_{l}^{i}(X)\right) \\
& \supset \operatorname{Hom}\left(V(-r), F_{b}^{r} H_{l}^{i}(X)\right) \ni f .
\end{aligned}
$$

(The last inclusion depends on the choice of stable complement for $V$ in $H_{l}^{i-2 r}(T)$.) As $f$ is fixed by $\operatorname{Gal}(\mathbb{F} / k)$, it can be approximated by the cohomology class of an algebraic correspondence $z$ of degree $r$ from $T$ to $X$. Moreover, $z$ can be chosen

\footnotetext{
${ }^{2}$ Recall that the push-forward $q_{*}(z)$ of an irreducible $z$ is defined to be zero if $\operatorname{dim}(q(z))<$ $\operatorname{dim} z$.
} 
so that $z_{*}$ is injective on $V$. Obviously $z_{*}$ maps $H_{l}^{i-2 r}(T)(-r)$ into $F_{b}^{r} H_{l}^{i}(X)$, and so

$$
F_{b}^{r} H_{l}^{i}(X) \subset z_{*} V(-r) \stackrel{1.8}{\subset} F_{a}^{r} H_{l}^{i}(X) .
$$

Theorem 1.10 Let $X$ be a smooth complete variety over $k$. If the Tate conjecture holds for all varieties of the form $A \times X$ with $A$ an abelian variety (and some $l$ ), then the generalized Tate conjecture holds for $X$ (and the same $l$ ).

Proof. As we noted above, $H_{l}^{*}(A)$ is a semisimple, and so this follows from (1.7) and 1.9).

COROLlary 1.11 If the Tate conjecture holds for all abelian varieties over $k$ (or for all smooth complete varieties over $k$ ) and some $l$, then the generalized Tate conjecture holds for the same class and that $l$.

REmark 1.12 As others have noted (Kahn 2002, Theorem 2; André 2004, 8.2), when one assumes the full Tate conjecture, the generalized Tate conjecture follows directly from the description of the simple motives in terms of Weil numbers (see Milne 1994, Proposition 2.6).

\section{Complements}

1.13 Let $X$ be a smooth projective variety over $k$, and let $V=F_{b}^{r} H_{l}^{i}(X)$. We know that $V(-r) \subset H_{l}^{i-2 r}(A)$ for some abelian variety $A$ over $k$ (see 1.7). If $\operatorname{dim} A=d>i-2 r$, then, according to the Lefschetz hypersurface-section theorem, for any smooth hypersurface section $Y$ of $A$ (which exists by Gabber 2001), $V(-r) \subset H_{l}^{i-2 r}(Y)$. Continuing in this fashion, we get that $V(-r) \subset$ $H_{l}^{i-2 r}(T)$ for some smooth projective $T$ of dimension $i-2 r$. Therefore, under the assumption of the Tate conjecture, there exists a smooth projective variety $T$ of dimension at most $i-2 r$ over $k$ and an algebraic correspondence $z$ from $T$ to $X$ of degree $r$ such that $z_{*} H_{l}^{i-2 r}(T)(r)=F_{b}^{r} H_{l}^{i}(X)$.

1.14 Deligne (1974b, 8.2.8) proves the following: 
Let $X$ be a smooth complete variety over $\mathbb{C}$, and let $Z$ be a closed subvariety of $X$ of codimension $r$. For any desingularization $\tilde{Z} \rightarrow Z$ of $Z$, the sequence

$$
H^{i-2 r}(\tilde{Z}, \mathbb{Q}(-r)) \rightarrow H^{i}(X, \mathbb{Q}) \rightarrow H^{i}(U, \mathbb{Q}), \quad U=X \backslash Z,
$$

is exact.

A similar argument ${ }^{3}$ proves the following $l$-adic analogue:

Let $X$ be a smooth complete variety over a perfect field $k$, and let $Z$ be a closed subvariety of $X$ of codimension $r$. For any smooth alteration $\tilde{Z} \rightarrow Z$ of $Z$, the sequence

$$
H_{l}^{i-2 r}(\tilde{Z})(-r) \rightarrow H_{l}^{i}(X) \rightarrow H_{l}^{i}(U), \quad U=X \backslash Z,
$$

is exact.

Since de Jong $(1996,3.1)$ shows that smooth alterations always exist, this implies that

$$
F_{a}^{r} H_{l}^{i}(X) \subset F_{b}^{r} H_{l}^{i}(X) .
$$

The generalized Tate conjecture then states that

$$
F_{a}^{r} H_{l}^{i}(X)=F_{b}^{r} H_{l}^{i}(X) .
$$

1.15 The above statements hold mutatis mutandis for $p$. For a smooth complete variety $X, H_{p}^{i}(X)$ is an $F$-isocrystal, i.e., a finite-dimensional vector space over $B\left(\mathbb{F}_{q}\right) \stackrel{\text { def }}{=} W\left(\mathbb{F}_{q}\right) \otimes \mathbb{Q}$ equipped with a $\sigma$-linear bijection $F: H_{p}^{i}(X) \rightarrow H_{p}^{i}(X)$. The full Tate conjecture for $X$ and $r$ is equivalent to

\footnotetext{
${ }^{3}$ For any proper surjective morphism $f: Y \rightarrow Z$ from a smooth projective variety $Y$, we can find a smooth projective simplicial scheme $Y_{\bullet}$ with $Y_{0}=Y$ that is a proper hypercovering of $Z$. The corresponding spectral sequence ( $l$-adic analogue of the spectral sequence Deligne 1974b, 8.1.19.1) degenerates at $E_{2}$ with $\mathbb{Q}_{l}$-coefficients because of weight considerations, and gives an exact sequence

$$
0 \rightarrow \frac{H_{l}^{i}(Z)}{W_{i-1} H_{l}^{i}(Z)} \rightarrow H_{l}^{i}\left(Y_{0}\right) \stackrel{\delta_{0}-\delta_{1}}{\longrightarrow} H_{l}^{i}\left(Y_{1}\right)
$$

It follows that the image of $H_{l}^{i}(Z)$ in $H_{l}^{i}(Y)$ is the (largest) quotient of pure weight $i$ of $H_{l}^{i}(Z)$. This implies the $l$-adic analogue of Deligne 1974b, 8.2.7, (the proof there works as the $G r_{*}^{W}$ functor is exact) and of ibid. 8.2.8.
} 
$T^{r}(X, p)$ : the cycle class map $Z^{r}(X) \otimes \mathbb{Q}_{p} \rightarrow H_{p}^{2 r}(X)(r)^{F=1}$ is surjective (Tate conjecture for $p$ ), and

$E^{r}(X, p)$ : the quotient map $Z_{\operatorname{hom}(p)}^{r}(X)_{\mathbb{Q}} \rightarrow Z_{\text {num }}^{r}(X)_{\mathbb{Q}}$ is injective

(cf. Milne 2007, §1). Define

$$
F_{a}^{r} H_{p}^{i}(X)=\bigcup_{Z} \operatorname{Im}\left(H_{p}^{i-2 r}(\tilde{Z})(-r) \rightarrow H_{p}^{i}(X)\right)
$$

where $Z$ runs over the closed subvarieties of $X$ such that $Z$ is of codimension at least $r$ and $\tilde{Z}$ is a smooth alteration of $Z$. If the Tate conjecture holds for smooth complete varieties over $k$ and $p$, then

$$
F_{a}^{r} H_{p}^{i}(X)=F_{b}^{r} H_{p}^{i}(X)
$$

where $F_{b}^{r} H_{p}^{i}(X) \subset H_{p}^{i}(X)_{[r, \infty)}$ is the largest semisimple sub-isocrystal of $H_{p}^{i}(X)$ with slopes at least $r$. The proofs are similar to those in the case $l \neq p$-we omit the details.

1.16 Similar arguments show that the generalized Tate conjecture over number fields follows from the Tate conjecture and an effective version of the FontaineMazur conjecture (Fontaine and Mazur 1995, Conjecture 1, p44) that specifies which representations arise from effective motives.

Notes It was known to Grothendieck that the generalized Hodge conjecture follows from the usual Hodge conjecture and the following weak analogue of (1.7),

Let $V$ be a simple Hodge substructure of the cohomology of a smooth complex projective variety; if its Tate twist $V(r)$ is still effective (i.e., has only nonnegative Hodge numbers), then $V(r)$ occurs in the cohomology of a smooth complex projective variety.

presumably by more-or-less the above argument. See Grothendieck 1969, top of p301 (also Schoen 1989, §0).

\section{The category of pure motives}

In this section $k=\mathbb{F}_{q}$. 
For any adequate equivalence relation $\sim$, Grothendieck's construction gives a rigid pseudo-abelian tensor $\mathbb{Q}$-category $\mathcal{M}_{\sim}(k)$ of pure motives (Saavedra Rivano 1972, VI 4.1.3.5, p359) and a map $h$ from the smooth projective varieties over $k$ to $\mathcal{M}_{\sim}(k)$ which is natural for algebraic correspondences modulo $\sim$. Because rational equivalence is the finest adequate equivalence relation, $h$ factors through a tensor functor $\mathcal{M}_{\text {rat }}(k) \rightarrow \mathcal{M}_{\sim}(k)$. Conversely, a tensor functor from $\mathcal{M}_{\text {rat }}(k)$ to an additive tensor category with $\operatorname{End}(\mathbb{1})=\mathbb{Q}$ defines an adequate equivalence relation (cf. Jannsen $2000,1.7$ ). When $\sim$ is numerical equivalence, $\mathcal{M}_{\sim}(k)$ is a semisimple (Jannsen 1992).

For a smooth projective variety $X$ over $k$, there are well-defined polynomials $P_{X, i}(T) \in \mathbb{Q}[T]$ such that $P_{X, i}(T)=\operatorname{det}\left(1-\varpi_{X} T \mid H_{l}^{i}(X)\right)$ for all $l$; moreover, $P_{X, i}$ has reciprocal roots of absolute value $q^{\frac{i}{2}}$ (Deligne 1974a). The $P_{X, i}(T)$ are relatively prime, and so there exist $P^{i}(T) \in \mathbb{Q}[T]$, well-defined up to a multiple of $\prod_{i} P_{X, i}(T)$, such that

$$
P^{i}(T) \equiv \begin{cases}1 & \bmod P_{X, i}(T) \\ 0 & \bmod P_{X, j}(T) \text { for } j \neq i .\end{cases}
$$

Because $\prod_{i} P_{X, i}\left(\varpi_{X}\right)$ acts as zero on $H_{l}^{*}(X)$, the graph $p^{i}$ of $P^{i}\left(\varpi_{X}\right)$ is a welldefined element of $Z_{\mathrm{hom}(l)}(X \times X)_{\mathbb{Q}}\left(\right.$ or $\left.Z_{\mathrm{num}}(X \times X)_{\mathbb{Q}}\right)$, and $\left\{p^{0}, \ldots, p^{2 d}\right\}$ is a complete set of orthogonal idempotents. Let $h X=\bigoplus_{i} h^{i} X$ be the corresponding decomposition. When we use this decomposition to modify the commutativity constraint in $\mathcal{M}_{\text {num }}(k)$, the rank of each object of $\mathcal{M}_{\text {num }}(k)$ becomes a nonnegative integer, and so $\mathcal{M}_{\text {num }}(k)$ is a tannakian category (Deligne 1990, 7.1).

The category $\mathcal{M}_{\text {num }}(k)$ has a canonical (Frobenius) element $\varpi \in \mathrm{Aut}^{\otimes}\left(\operatorname{id}_{\mathcal{M}_{\text {num }}(k)}\right)$ and a canonical (weight) $\mathbb{Z}$-gradation. An object $M$ of $\mathcal{M}_{\text {num }}(k)$ is of pure weight $m$ if and only if its Frobenius element $\varpi_{M}$ has eigenvalues of absolute value $q^{m / 2}$.

Recall (Deligne 1989, §6) that the fundamental group $\pi(\mathcal{T})$ of a tannakian category is an affine group scheme in Ind $\mathcal{T}$ that acts on each object of $\mathcal{T}$ in such a way that these actions define an isomorphism

$$
\omega(\pi(\mathcal{T})) \simeq \underline{\operatorname{Aut}}^{\otimes}(\omega)
$$

for each fibre functor $\omega$. Any subgroup of the centre of $\pi(\mathcal{T})$ lies in $\operatorname{Ind} \mathcal{T}^{0}$ where $\mathcal{T}^{0}$ is the full subcategory of trivial objects (those isomorphic to a multiple of $\mathbb{1}$ ). 
Since $\operatorname{Hom}_{\mathcal{T}}(\mathbb{1},-)$ defines an equivalence of $\mathcal{T}^{0}$ with the finite-dimensional vector spaces over the ground field, such a subgroup can be identified with an affine group scheme in the usual sense. For example, the centre of $\pi(\mathcal{T})$ is $\underline{A u t}^{\otimes}(\operatorname{id} \mathcal{T})$ (cf. Saavedra Rivano 1972, II 3.3.3.2).

Recall (e.g., Milne 1994, §2) that the Weil-number group $P$ is the affine group scheme of multiplicative type over $\mathbb{Q}$ whose character group consists of the Weil $q$-numbers in $\mathbb{Q}^{\text {al }}$. Define the Frobenius element $\varpi_{\text {univ }}$ in $P(\mathbb{Q})$ to be that corresponding to $\alpha \mapsto \alpha$ under the bijection

$$
P(\mathbb{Q}) \simeq \operatorname{Hom}\left(X^{*}(P), \mathbb{Q}^{\mathrm{al}}\right)^{\operatorname{Gal}\left(\mathbb{Q}^{\mathrm{al}} / \mathbb{Q}\right)} .
$$

Note that, for any smooth projective variety $X$ over $\mathbb{F}_{q}$, the roots of $P_{X, i}(T)$ in $\mathbb{Q}^{\text {al }}$ are Weil $q$-integers of weight $i$ (i.e., Weil $q$-numbers of weight $i$ that are algebraic integers).

Lemma 2.1 The group of Weil q-numbers is generated by the Weil q-numbers of abelian varieties over $k$.

Proof. Let $\alpha$ be a Weil $q$-number. After multiplying $\alpha$ by a power of $q$, we may suppose that it is a Weil $q$-integer, of weight $m$ say. Then $\alpha^{1 / m}$ is a Weil $q$-integer of weight 1, and hence arises from an abelian variety by Honda 1968.

Proposition 2.2 The affine subgroup scheme of $\pi\left(\mathcal{M}_{\text {num }}(k)\right)$ generated by $\varpi_{\text {univ }}$ is canonically isomorphic to $P$. It equals $\pi\left(\mathcal{M}_{\text {num }}(k)\right)$ if and only if the full Tate conjecture holds over $k$.

Proof. Let $Z={\underline{\operatorname{Aut}^{\otimes}}}^{\otimes}(\mathrm{id})$ be the centre of $\pi\left(\mathcal{M}_{\text {num }}(k)\right)$. Because $\mathcal{M}_{\text {num }}(k)$ is semisimple, $\pi\left(\mathcal{M}_{\text {num }}(k)\right)$ is pro-reductive (cf. Deligne and Milne 1982, 2.23). Therefore $Z$ is of multiplicative type, which implies that the closed subgroup scheme $\left\langle\varpi_{\text {univ }}\right\rangle$ generated by $\varpi_{\text {univ }}$ is also of multiplicative type. The homomorphism $\chi \mapsto \chi\left(\varpi_{\text {univ }}\right): X^{*}\left(\left\langle\varpi_{\text {univ }}\right\rangle\right) \rightarrow \mathbb{Q}^{\text {al } \times}$ is injective, and its image consists of the Weil $q$-numbers that occur as roots of the characteristic polynomial of $\varpi_{M}$ for some $M$ in $\mathcal{M}_{\text {num }}(k)$. According to Lemma 2.1, this consists of all Weil $q$-numbers, and so $X^{*}\left(\left\langle\varpi_{\text {univ }}\right\rangle\right) \simeq X^{*}(P)$. Hence $\left\langle\varpi_{\text {univ }}\right\rangle \simeq P$.

If the full Tate conjecture holds, then, for any fibre functor $\omega$ over $\mathbb{Q}^{\text {al }}$ and smooth projective variety $X$, the $\mathbb{Q}^{\text {al }}$-span of the algebraic cycles in $\omega\left(h^{2 i}(X)(i)\right)$ 
consists of the tensors fixed by $\varpi_{\text {univ }}$. Therefore, the inclusion $\left\langle\varpi_{\text {univ }}\right\rangle \hookrightarrow \underline{\text { Aut }}^{\otimes}(\omega)$ is an isomorphism, i.e., $\omega(P) \hookrightarrow \omega\left(\pi\left(\mathcal{M}_{\text {num }}(k)\right)\right.$ is an isomorphism, which implies that $P \hookrightarrow \pi\left(\mathcal{M}_{\text {num }}(k)\right)$ is an isomorphism. The converse can be proved by the same argument as in the proof of Milne 1999, Proposition 7.4.

If num and $\operatorname{hom}(l)$ coincide with $\mathbb{Q}$-coefficients, then $H_{l}$ defines a fibre functor $\omega_{l}$ on $\mathcal{M}_{\text {num }}(k)$. Without any assumptions, it is known that there exists a polarizable semisimple tannakian category with fundamental group $P$ and with fibre functors $\omega_{l}$ for all $l$. Moreover, any two such systems are equivalent (Langlands and Rapoport 1987; Milne 2003, §6). However, it has not been shown that there exists a natural functor from $\mathcal{M}_{\text {rat }}(k)$ to such category. In fact, we have the following:

Proposition 2.3 If there exists a full tensor functor $r$ preserving Frobenius elements from $\mathcal{M}_{\text {rat }}(k)$ to a tannakian category $\mathcal{M}$ with fundamental group $P$, then the full Tate conjecture holds over $k$, and $r$ defines an equivalence of tensor categories $\mathcal{M}_{\text {num }}(k) \rightarrow \mathcal{M}$.

PROOF. Such a functor $r$ defines an adequate equivalence relation $\sim$ (see above) such that $r$ factors into

$$
\mathcal{M}_{\text {rat }}(k) \rightarrow \mathcal{M}_{\sim}(k) \stackrel{\bar{r}}{\longrightarrow} \mathcal{M}
$$

with $\bar{r}$ a fully faithful tensor functor. Because $P$ is a pro-reductive group, $\mathcal{M}$ is semisimple (cf. Deligne and Milne 1982, 2.23). It follows that $\mathcal{M}_{\sim}(k)$ is semisimple (apply the criterion in Jannsen 1992, Lemma 2), and so $\sim$ is numerical equivalence (ibid. Theorem 1). The simple objects of $\mathcal{M}$ are classified by the orbits of $\operatorname{Gal}\left(\mathbb{Q}^{\text {al }} / \mathbb{Q}\right)$ acting on $X^{*}(P)$, i.e., by the conjugacy classes of Weil $q$-numbers, and so Lemma 2.1 shows that $\mathcal{M}$ is generated as a tensor category by the images of abelian varieties. Therefore, $\bar{r}$ is a tensor equivalence, and so defines an isomorphism of $P$ with $\pi\left(\mathcal{M}_{\text {num }}(k)\right)$. We can now apply Proposition 2.2.

REMARK 2.4 When we drop the requirement that $r$ is full, then it is possible to work with hypotheses much weaker than the full Tate conjecture. Let $\mathcal{S}$ consist of the smooth projective varieties over $\mathbb{F}_{q}$ whose Frobenius elements are semisimple. In Milne 2007 a notion of a "good theory of rational Tate classes on $\mathcal{S}$ " is defined, 
and it is proved that there exists at most one such theory. Much of this paper could be rewritten with "full Tate conjecture" replaced by "there exists a good theory of rational Tate classes for which the algebraic classes are rational Tate" provided one removes the requirement that certain functors are full.

\section{The category of motives}

The next observation goes back to Grothendieck.

Proposition 3.1 Let $\mathcal{M M}\left(\mathbb{F}_{q}\right)$ be a pseudo-abelian category containing $\mathcal{M}_{\text {num }}\left(\mathbb{F}_{q}\right)$ as a full subcategory. Assume

(a) each object $M$ of $\mathcal{M M}\left(\mathbb{F}_{q}\right)$ has a (weight) filtration

$$
\cdots \subset W_{i-1} M \subset W_{i} M \subset \cdots
$$

such that $W_{i} M / W_{i-1} M$ is a pure motive of weight $i$;

(b) the Frobenius element extends to $\mathcal{M M}\left(\mathbb{F}_{q}\right)$ and preserves the weight filtrations.

Then the inclusion $\mathcal{M}_{\text {num }}\left(\mathbb{F}_{q}\right) \rightarrow \mathcal{M M}\left(\mathbb{F}_{q}\right)$ is an equivalence of tensor categories.

Proof. For $X$ in $\mathcal{M M}\left(\mathbb{F}_{q}\right)$, let $P_{i}(T)$ be the characteristic polynomial of $\varpi_{W_{i} M / W_{i-1} M}$, and define $P^{i}(T)$ to satisfy $(2)$. Let $p^{i}=P^{i}\left(\varpi_{M}\right)$. Then the $p^{i}$ form a complete set of orthogonal idempotents in $\operatorname{End}(M)$ which decompose $M$ into a direct sum isomorphic to $\bigoplus_{i} W_{i} M / W_{i-1} M$.

\section{Triangulated motivic categories}

Recall that a tensor triangulated category is a category with both a tensor structure and a triangulated structure satisfying certain compatibilities (Mazza et al. 2006, Appendix 8A). It is rigid if it admits an internal Hom or, equivalently, a good theory of duals (Voevodsky 2000, p196). By a triangulated motivic category over a field $k$, we mean a rigid tensor triangulated category $\mathcal{D}$ together with a covariant functor

$$
R: \mathcal{M}_{\text {rat }}(k) \rightarrow \mathcal{D}
$$


and isomorphisms for all smooth projective varieties $X$ and all $i, j \in \mathbb{Z}$

$$
K_{2 j-i}(X)^{(j)} \longrightarrow \operatorname{Hom}_{\mathcal{D}}(\mathbb{1}, R(h X)(j)[i])
$$

that are natural for the maps defined by algebraic correspondences and reduce to the identity map when $X$ is a point and $i=j=0$ (see Jannsen 2000, $\S 7$, p257, which omits the final condition). Here $K_{i}(X)^{(j)}$ is the subspace of $K_{i}(X) \otimes \mathbb{Q}$ on which each Adams operator $\psi^{m}$ acts as $m^{j}$. According to ibid., p257, over any field $k$ that admits resolution of singularities, triangulated motivic categories have been constructed (independently) by Hanamura (1995, 1999, 2004), Levine (1998), and Voevodsky (2000). When $k=\mathbb{F}_{q}, \psi^{q}$ acts as $\varpi_{X}$ (Hiller 1981, §5; Soulé $1985,8.1)$, and $\operatorname{so}^{4} K_{i}(X)^{(j)}$ is the subspace on which $\varpi_{X}$ acts as $q^{j}$.

Let $\mathcal{D}=\mathcal{D}(k)$ be a triangulated motivic category. As we noted in the introduction, for the "true" triangulated motivic category, there should be a $t$ structure on $\mathcal{D}(k)$ whose heart $\mathcal{M} \mathcal{M}(k) \stackrel{\text { def }}{=} \mathcal{D}(k)^{\bigcirc}$ is the category of mixed motives. As Jannsen $(2000, \S 7$, p257) explains, there should be the following compatibilities between $R$ and the $t$-structure:

(a) for each standard Weil cohomology, the composite

$$
\begin{aligned}
\mathcal{M}_{\mathrm{rat}}(k) \stackrel{R}{\longrightarrow} \mathcal{D} \stackrel{\oplus_{i} H^{i}}{\longrightarrow} \mathcal{M M}(k) \\
K \mapsto \bigoplus_{i} H^{i}(K)
\end{aligned}
$$

factors through $\mathcal{M}_{\text {hom }}(k)$, and defines a fully faithful functor $\bar{R}: \mathcal{M}_{\text {hom }}(k) \rightarrow$ $\mathcal{M M}(k)$ (here $\left.H^{i}(K)=\tau_{\leq 0} \tau_{\geq 0}(K[-i])\right) ;$

(b) for each smooth projective variety $X, \bigoplus_{i} H^{i}(R(h X))$ is the weight gradation of $h X$.

When $k$ is finite, condition (b) says that $H^{i}(R(h X))=\bar{R}\left(h^{i}(X)\right)$.

Evidently, there should also be the following compatibilities between the tensor structures and the $t$-structure:

(c) the subcategories $\mathcal{D} \leq 0$ and $\mathcal{D}^{\geq 0}$ are tensor subcategories of $\mathcal{D}$, and $M \mapsto M^{\vee}$ interchanges $\mathcal{D} \leq 0$ and $\mathcal{D} \geq 0$, and

(d) $R: \mathcal{M}_{\text {rat }}(k) \rightarrow \mathcal{M M}(k)$ is a tensor functor.

\footnotetext{
${ }^{4}$ Because the $m^{i}$-eigenspace of $\psi^{m}$ is independent of $m$ (Seiler 1988, Theorem 1).
} 
Note that (c) implies that $\mathcal{M M}(k)$ is a rigid tensor subcategory of $\mathcal{D}$.

Definition $4.1 \mathrm{~A} t$-structure on a triangulated motivic category is said to be admissible if it satisfies the conditions (a,b,c,d).

Theorem 4.2 Let $k$ be a finite field. If there exists a triangulated motivic category $\mathcal{D}$ over $k$ and an admissible $t$-structure on $\mathcal{D}$ such that

- the heart of $\mathcal{D}$ is a tannakian category $\mathcal{M}$ with fundamental group $P$, and

- the functor $\mathcal{M}_{\text {rat }}(k) \rightarrow \mathcal{M}$ in (a) above preserves Frobenius elements,

then

(a) the full Tate conjecture holds for all smooth projective varieties over $k$;

(b) for each $l$, the functor $R_{l}: \mathcal{M}_{\text {hom(l) }}(k) \rightarrow \mathcal{M}$ defined by $R$ is an equivalence of abelian categories;

(c) rational equivalence equals numerical equivalence ( $\mathbb{Q}$-coefficients);

(d) for all $M, N$ in $\mathcal{M}(k)$ and $i \neq 0, \operatorname{Hom}_{\mathcal{D}}(M, N[i])=0$.

Proof. Proposition 2.3 shows that the full Tate conjecture holds and that $R_{l}$ is essentially surjective (hence an equivalence). Moreover, it allows us to identify $\mathcal{M}$ with $\mathcal{M}_{\text {num }}(k)$.

We next prove (c). When $i=2 j$, the isomorphism (3) becomes

$$
K_{0}(X)^{(j)} \simeq \operatorname{Hom}_{\mathcal{D}}(\mathbb{1}, R(h X)(j)[2 j]) .
$$

As we noted above, $\varpi_{X}$ acts on $K_{0}(X)^{(j)}$ as $q^{j}$. The Tate conjecture implies the Lefschetz standard conjecture, and so, for any smooth projective variety $X$, there exists an isomorphism

$$
R(h X)(j)[2 j] \approx \bigoplus_{s} h^{s}(X)(j)[2 j-s]
$$

(Deligne 1968, Van den Bergh 2004). The characteristic polynomial $P_{X, s}$ of $\varpi_{X}$ on $h^{s} X$ has roots of absolute value $q^{s / 2}$, and $P_{X, s}\left(\varpi_{X}\right)$ acts as zero on $h^{s}(X)$ and hence on $\operatorname{Hom}_{\mathcal{D}}\left(\mathbb{1}, h^{s}(X)(j)[2 j-s]\right)$. But we know from (4) that it acts as $P_{X, s}\left(q^{j}\right)$. Therefore, $\operatorname{Hom}_{\mathcal{D}}\left(\mathbb{1}, h^{s}(X)(j)[2 j-s]\right)=0$ unless $s=2 j$, and so (4) becomes

$$
K_{0}(X)^{(j)} \simeq \operatorname{Hom}_{\mathcal{M}_{\text {num }}(k)}\left(\mathbb{1}, h^{2 j}(X)(j)\right)
$$


Under Grothendieck's isomorphism $K_{0}(X)_{\mathbb{Q}} \simeq C H^{*}(X)_{\mathbb{Q}}$, the factors $K_{0}(X)^{(j)}$ and $C H^{j}(X)_{\mathbb{Q}}$ correspond (this is obvious over a finite field), and (by definition)

$$
\operatorname{Hom}_{\mathcal{M}_{\text {num }}(k)}\left(\mathbb{1}, h^{2 j}(X)(j)\right)=Z_{\text {num }}^{j}(X)_{\mathbb{Q}}
$$

Moreover, our conditions imply that the isomorphism

$$
C H^{j}(X)_{\mathbb{Q}} \simeq Z_{\text {num }}^{j}(X)_{\mathbb{Q}}
$$

obtained by combining these isomorphisms is the canonical one. ${ }^{5}$ Hence, we have proved (c), and we have shown that

$$
\operatorname{Hom}_{\mathcal{D}}(\mathbb{1}, R(h X)(j)[i])=0
$$

when $i=2 j \neq 0$.

Finally, we prove (d). Because $\mathcal{M}$ is a rigid subcategory of $\mathcal{D}$, for $M, N$ in $\mathcal{M}$ there exists an object $\underline{\operatorname{Hom}}(M, N)$ in $\mathcal{M}$ such that $\operatorname{Hom}_{\mathcal{D}}(T \otimes M, N) \simeq$ $\operatorname{Hom}_{\mathcal{D}}(T, \underline{\operatorname{Hom}}(M, N))$ for all $T$ in $\mathcal{D}$. In particular,

$$
\operatorname{Hom}_{\mathcal{D}}(M, N[i]) \simeq \operatorname{Hom}_{\mathcal{D}}(\mathbb{1}, \underline{\operatorname{Hom}}(M, N)[i]) .
$$

Therefore, because every object of $\mathcal{M}$ is a direct summand of $R(h X)(j)$ for some smooth projective variety $X$ and integer $j$, it suffices to prove (d) with $M=\mathbb{1}$ and $N=R(h X)(j)$. We know it when $i=2 j$ (see (7)), and so, to

${ }^{5}$ Let $p$ and $q$ be the projection maps

$$
\text { pt } \stackrel{p}{\longleftarrow} X \times \text { pt } \stackrel{q}{\longrightarrow} X .
$$

Let $\gamma \in C H^{j}(X)$, and let $f$ be the map $C H^{*}(\mathrm{pt}) \rightarrow C H^{*+j}(X)$ defined by the correspondence $q^{*}(\gamma)$. Then

$$
f\left(1_{\mathrm{pt}}\right) \stackrel{\text { def }}{=} q_{*}\left(q^{*}(\gamma) \cup p^{*}\left(1_{\mathrm{pt}}\right)\right)=\gamma \cup q_{*} p^{*}\left(1_{\mathrm{pt}}\right)=\gamma \cup 1_{X}=\gamma .
$$

As (6) is functorial for correspondences, and the bottom row in

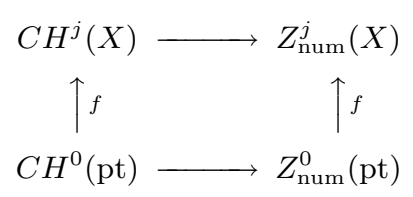

sends 1 to 1 (by assumption), it follows that the top row sends $\gamma$ to $\gamma$. 
complete the proof of (d), it remains to prove (7) when $i \neq 2 j$. Because of (3), it suffices to show that (a) and (c) imply that $K_{i}(X)_{\mathbb{Q}}=0$ whenever $i \neq 0$. This is done in Geisser 1998, 3.3. We recall the proof. The functors $K_{i}(X) \otimes \mathbb{Q}$ factor through $\mathcal{M}_{\text {rat }}(k)$ (Soulé 1984), and hence (because of (c)) through $\mathcal{M}_{\text {num }}(k)$. Therefore, it suffices to prove that $K_{i}(M) \otimes \mathbb{Q}=0(i \neq 0)$ for $M$ a simple motive in $\mathcal{M}_{\text {num }}(k)$. If $M=\mathbb{L}^{j}$, then $K_{i}\left(\mathbb{L}^{j}\right)$ is a direct factor of $K_{i}\left(\mathbb{P}^{j}\right)$, which is torsion (Quillen 1973). If $M \neq \mathbb{L}^{j}$, then $P_{M}(T)$ does not have $q^{j}$ as a root (Milne 1994, 2.6). As $P_{M}\left(\varpi_{X}\right)$ acts as the nonzero rational number $P_{M}\left(q^{j}\right)$ on $K_{i}(M)^{(j)}$, and also as zero, the group $K_{i}(M)^{(j)}$ must be zero.

Corollary 4.3 Let $\mathcal{D}$ be as in the theorem, and let $\mathcal{M}$ be its heart. If the inclusion $\mathcal{M} \rightarrow \mathcal{D}$ extends to a functor $\mathcal{D}^{b}(\mathcal{M}) \rightarrow \mathcal{D}$ (e.g., if $\mathcal{D}$ is endowed with a filtered triangulated category; see $4.6 \mathrm{a}$ below), then that functor is an equivalence.

Proof. It suffices to show that $\operatorname{Hom}_{\mathcal{D}^{b}(\mathcal{M})}(M, N[i]) \rightarrow \operatorname{Hom}_{\mathcal{D}}(M, N[i])$ is an isomorphism for all $M, N$ in $\mathcal{M}$ and all $i$ (see 4.6b below). For $i=0$ this is automatic, and for $i \neq 0$, both groups are zero (recall that $\operatorname{Hom}_{\mathcal{D}^{b}(\mathcal{M})}(M, N[i]) \simeq$ $\operatorname{Ext}_{\mathcal{M}}^{i}(M, N)$, and that $\mathcal{M}$ is semisimple).

REMARK 4.4 The existence of an admissible $t$-structure on a triangulated motivic category $\mathcal{D}$ implies the existence of a Bloch-Beilinson filtration on the Chow groups of smooth projective varieties for which

$$
G r^{s}\left(C H^{j}(X)\right) \simeq \operatorname{Hom}_{\mathcal{D}}\left(\mathbb{1}, h^{2 j-s}(X)(j)[s]\right)
$$

(Jannsen 2000, p258, 4.3). For a finite field, the existence of a Bloch-Beilinson filtration implies that rational equivalence equals numerical equivalence $(\mathbb{Q}$-coefficients) (ibid., 4.17).

REMARK 4.5 Beilinson has conjectured that, for a smooth projective variety $X$,

$$
G r^{s}\left(C H^{j}(X)\right)=\operatorname{Ext}_{\mathcal{M} \mathcal{M}_{\text {num }}(k)}\left(\mathbb{1}, h^{2 j-s}(X)(j)\right) .
$$

This is compatible with (8) only if $\mathcal{D}=\mathcal{D}^{b}\left(\mathcal{M M}_{\text {num }}(k)\right)$ (see the next remark).

REMARK 4.6 (a) Let $\mathcal{D}$ be a $t$-category with heart $\mathcal{C}$. Then $D^{b}(\mathcal{C})$ is also a $t$ category with heart $\mathcal{C}$, but in general there is no obvious relation between $D^{b}(\mathcal{C})$ 
and $\mathcal{D}$ (cf. Gelfand and Manin 1996, IV 4.13, p285). In particular, there will be no obvious functor $r: D^{b}(\mathcal{C}) \rightarrow \mathcal{D}$ extending the inclusion of $\mathcal{C}$ into $\mathcal{D}$ unless $\mathcal{D}$ is endowed with an additional structure. Beilinson (1987) defines the notion of a filtered triangulated category, and states ${ }^{6}$ that such a category over a $t$-category $\mathcal{D}$ gives rise to a well-defined $t$-exact functor $r: D^{b}(\mathcal{C}) \rightarrow \mathcal{D}$ inducing the identity functor on $\mathcal{C}$ (ibid. A.6). The usual triangulated categories are endowed with filtered triangulated categories over them (ibid. A.2; Beilinson et al. 1982, 3.1).

(b) Let $\mathcal{D}$ be a $t$-category with heart $\mathcal{C}$. A $t$-exact functor $r: D^{b}(\mathcal{C}) \rightarrow \mathcal{D}$ inducing the identity functor on $\mathcal{C}$ need not be an equivalence even when $\mathcal{C}$ is semisimple (Deligne 1994, 3.1). We need the following well-known criterion:

Let $r: D^{b}(\mathcal{C}) \rightarrow \mathcal{D}$ be a $t$-exact functor inducing the identity functor on $\mathcal{C}$; then $r$ is an equivalence of $t$-categories if and only if the maps $\operatorname{Hom}_{D^{b}(\mathcal{C})}(M, N[i]) \rightarrow \operatorname{Hom}_{\mathcal{D}_{\mathcal{C}}^{b}}(M, N[i])$ it defines are isomorphisms for all $M, N$ in $\mathcal{C}$ and all $i$.

For $M, N$ in $\mathcal{C}$, let $\operatorname{Ext}_{\mathcal{C}}^{i}(M, N)$ denote the Yoneda Ext-group, and for $M, N$ in the heart of $\mathcal{D}$, let

$$
\operatorname{Ext}_{\mathcal{D}}^{i}(M, N)=\operatorname{Hom}_{\mathcal{D}}(M, N[i]) .
$$

Since $\operatorname{Ext}_{\mathcal{C}}^{i}(M, N) \simeq \operatorname{Hom}_{D^{b}(\mathcal{C})}(M, N[i])$ (Verdier 1996, III.3.2.12), the criterion states that $r: D^{b}(\mathcal{C}) \rightarrow \mathcal{D}$ is an equivalence of $t$-categories if and only if the maps $\operatorname{Ext}_{\mathcal{C}}^{i}(M, N) \rightarrow \operatorname{Ext}_{\mathcal{D}}^{i}(M, N)$ it defines are isomorphisms for all $M, N$, and $i$.

\section{The motivic $t$-category}

Throughout this section, $k=\mathbb{F}_{q}$.

If we want the category of motives to have the Weil-number group $P$ as its fundamental group, then Corollary 4.3 shows that $\mathcal{D}^{b}\left(\mathcal{M}_{\text {num }}(k)\right)$ is essentially the only candidate for a triangulated motivic category, and that it will have

\footnotetext{
${ }^{6}$ Without proof; cf. the discussion Beilinson et al. 1982, 3.1, which, however, states that (at that time) the situation had not been axiomatised. Added in proof: see also J. Wildeshaus, f-categories and Tate motives, arXiv:0810.1674
} 
an admissible $t$-structure only if the Tate conjecture holds over $k$ and rational equivalence equals numerical equivalence ( $\mathbb{Q}$-coefficients). In this section, we prove that, when we assume these two conjectures, $\mathcal{D}^{b}\left(\mathcal{M}_{\text {num }}(k)\right)$ does have the hoped for properties.

Proposition 5.1 Let $\mathcal{D}=D^{b}\left(\mathcal{M}_{\text {num }}(k)\right)$. Then $\mathcal{D}$ is a rigid tensor triangulated category with $t$-structure, and there exists a tensor functor

$$
R: \mathcal{M}_{\text {rat }}(k) \rightarrow \mathcal{D}
$$

unique up to a unique isomorphism, such that $H^{i}(R X)=h^{i}(X)[-i]$ for all $i$.

Proof. Let $C^{b}\left(\mathcal{M}_{\text {num }}(k)\right)$ be the category of bounded complexes of objects in $\mathcal{M}_{\text {num }}(k)$, and let $C_{0}^{b}\left(\mathcal{M}_{\text {num }}(k)\right)$ be the full subcategory of bounded complexes whose differentials are zero. Because $\mathcal{M}_{\text {num }}(k)$ is semisimple, the functor $D^{b}(\mathcal{M}(k)) \rightarrow C_{0}^{b}\left(\mathcal{M}_{\text {num }}(k)\right)$ sending $A$ to

$$
\bigoplus_{r} H^{r}(A)[-r]=\cdots \rightarrow H^{r-1}(A) \stackrel{0}{\longrightarrow} H^{r}(A) \rightarrow \cdots
$$

is an equivalence of categories which is quasi-inverse to the inclusion functor (Gelfand and Manin 1996, III 2.4, p146). Since $C_{0}^{b}\left(\mathcal{M}_{\text {num }}(k)\right.$ ) is a direct sum of copies of $\mathcal{M}_{\text {num }}(k)$, and $\mathcal{M}_{\text {num }}(k)$ is tannakian, it follows that $\mathcal{D}$ is a rigid tensor category. Define $R$ to be

$$
X \mapsto\left(\cdots \rightarrow h^{r-1}(X) \stackrel{0}{\longrightarrow} h^{r}(X) \rightarrow \cdots\right)
$$

The uniqueness is obvious.

REMARK 5.2 Deligne $(1968,1.11,1.13)^{7}$ proves the following:

Let $\mathcal{A}$ be an abelian category, and suppose that an object $C$ of $D^{b}(\mathcal{A})$ admits endomorphisms $p_{i}: C \rightarrow C$ such that $H^{j}\left(p_{i}\right)=\delta_{i j}$ and the $p_{i}$ are orthogonal idempotents; then there is a unique isomorphism $C \simeq \bigoplus_{i} H^{i}(C)[-i]$ inducing the identity map on cohomology and such that $p_{i}$ is the $i^{\text {th }}$ projection map.

\footnotetext{
${ }^{7}$ This also applies to $t$-categories. To check this, one only has to check that the spectral sequence in Deligne's proof exists for $t$-categories (for which there exist references).
} 
Let $R^{\prime}$ be a tensor functor $\mathcal{M}_{\text {rat }}(k) \rightarrow \mathcal{D}^{b}\left(\mathcal{M}_{\text {num }}(k)\right)$ and let $R$ be as in (9). Then Deligne's result shows that, for any smooth projective variety $X$ over $k$, there is a unique isomorphism $R^{\prime}(X) \simeq R(X)$ inducing the identity on cohomology and such that $P^{i}\left(\varpi_{X}\right)$ is the projection from $R^{\prime}(X)$ onto $h^{i}(X)[-i]$. Here $P^{i}$ is as in (2).

Theorem 5.3 Assume that the Tate conjecture holds over $k$ and that numerical equivalence coincides with rational equivalence (with $\mathbb{Q}$-coefficients).

(a) $D^{b}\left(\mathcal{M}_{\text {num }}(k)\right)$ has a natural structure of a triangulated motivic category.

(b) The standard t-structure on $D^{b}\left(\mathcal{M}_{\text {num }}(k)\right)$ is admissible (in the sense of $\S 4)$, and it is the unique t-structure on $D^{b}\left(\mathcal{M}_{\text {num }}(k)\right)$ with heart $\mathcal{M}_{\text {num }}(k)$.

(c) The functor $X \mapsto R X$ sending a smooth projective variety over $k$ to its motivic complex (see 5.1) has a unique extension to all varieties over $k$.

(d) For each $l$ (including $p$ ) there is a $t$-exact functor $R_{l}$ from $D^{b}\left(\mathcal{M}_{\text {num }}(k)\right)$ to a $t$-category $\mathcal{D}_{l}$ such that $X \mapsto R_{l}(R X)$ is the functor giving rise to the absolute l-adic cohomology.

In the remainder of this section, we explain these statements in more detail and prove them.

Statement (a). We have to construct isomorphisms (3). In computing the right hand side of $(3)$, we can replace $\mathcal{D}^{b}\left(\mathcal{M}_{\text {num }}(k)\right)$ with the equivalent category $C_{0}^{b}\left(\mathcal{M}_{\text {num }}(k)\right) \simeq \bigoplus_{r} \mathcal{M}_{\text {num }}(k)[r]$. Therefore,

$$
\operatorname{Hom}_{\mathcal{D}}(\mathbb{1}, R(X)(j)[i])=\bigoplus_{s} \operatorname{Hom}_{\mathcal{D}}\left(\mathbb{1}, h^{s}(X)(j)[i-s]\right),
$$

and

$$
\operatorname{Hom}_{\mathcal{D}}\left(\mathbb{1}, h^{s}(X)(j)[i-s]\right)=\operatorname{Ext}_{\mathcal{M}_{\text {num }}(k)}^{i-s}\left(\mathbb{1}, h^{s}(X)(j)\right) .
$$

This group is zero for $i \neq s$ because $\mathcal{M}_{\text {num }}(k)$ is semisimple, and it is zero for $i=s, s \neq 2 j$, because $\mathbb{1}$ and $h^{s}(X)(j)$ will then have different weights. It is immediate from the definition of $\mathcal{M}_{\text {num }}(k)$, that

$$
\operatorname{Hom}\left(\mathbb{1}, h^{2 j}(X)(j)\right) \simeq Z_{\text {num }}^{j}(X)_{\mathbb{Q}} .
$$

On the other hand, $K_{i}(X)_{\mathbb{Q}}=0$ for $i \neq 0$ (see the proof 4.2$)$, and $K_{0}(X)^{(j)} \simeq$ $C H^{j}(X)_{\mathbb{Q}}$. Therefore, we can define (3) to be the natural map

$$
C H^{j}(X)_{\mathbb{Q}} \rightarrow Z_{\text {num }}^{j}(X)_{\mathbb{Q}}
$$


when $i=2 j$ and zero otherwise.

Statement (b). By hypothesis, rational, l-homological, and numerical equivalence coincide ( $\mathbb{Q}$-coefficients), and so the standard $t$-structure is obviously admissible. It is the unique $t$-structure with heart $\mathcal{M}_{\text {num }}(k)$ because the heart determines the $t$-structure (Beilinson et al. 1982, 1.2, 1.3).

Statement (c). We only sketch the argument, leaving the details as an exercise for the reader. The key point is that de Jong 1996, Theorem 3.1, allows one to define a simplicial resolution

$$
V \stackrel{f}{\longleftarrow} U_{\bullet} \stackrel{j}{\longrightarrow} X \bullet
$$

of any variety $V$ over $k$ in which $j$ is a simplicial strict compactification and $f$ is a proper hypercovering of $V$ by a split simplicial smooth variety (cf. Berthelot 1997, 6.3). One first extends $R$ to the category of strict compactifications, and then to the simplicial objects in the category of strict compactifications. Then one defines $R V=R\left(U_{\bullet} \rightarrow X_{\bullet}\right)$ for any simplicial resolution $V \stackrel{f}{\longleftarrow} U_{\bullet} \stackrel{j}{\longrightarrow} X_{\bullet}$ of $V$. One verifies that $R V$ is independent of the choice of the simplicial resolution (up to a well-defined isomorphism), and the map $V \mapsto R V$ is contravariant for morphisms of varieties.

Statement $(\mathbf{d}), l \neq p$. For $l \neq p$, let $D_{c}^{b}\left(k, \mathbb{Z}_{l}\right)$ be the category defined in Deligne $1980,1.1 .2$. It is a $t$-category whose heart is the category $\mathcal{R}\left(k, \mathbb{Z}_{l}\right)$ of finitely generated $\mathbb{Z}_{l}$-modules endowed with a continuous action of $\operatorname{Gal}(\mathbb{F} / k)$. Each variety $V$ over $k$ defines an object $R \Gamma V$ in $D_{c}^{b}\left(k, \mathbb{Z}_{l}\right)$ such that $H^{i}(R \Gamma V) \simeq$ $H_{\text {et }}^{i}\left(V, \mathbb{Z}_{l}\right)$ (as objects of $\mathcal{R}\left(k, \mathbb{Z}_{l}\right)$ ). It is known that $D_{c}^{b}\left(k, \mathbb{Z}_{l}\right) \simeq D^{b}\left(\mathcal{R}\left(k, \mathbb{Z}_{l}\right)\right.$ ). Now quotient out by the torsion objects to obtain an equivalence $D_{c}^{b}\left(k, \mathbb{Q}_{l}\right) \simeq$ $D^{b}\left(\mathcal{R}\left(k, \mathbb{Q}_{l}\right)\right)$ of $\mathbb{Q}_{l}$-linear categories. We define

$$
R_{l}: D^{b}\left(\mathcal{M}_{\mathrm{num}}(k)\right) \rightarrow D^{b}\left(\mathcal{R}\left(k, \mathbb{Q}_{l}\right)\right) \simeq D_{c}^{b}\left(k, \mathbb{Q}_{l}\right)
$$

to be the derived functor of the fibre functor $\mathcal{M}_{\text {num }}(k) \rightarrow \mathcal{R}\left(k, \mathbb{Q}_{l}\right)$. Applying Deligne 1968, 1.11, 1.13 (cf. 5.2), we see that, for each smooth projective variety $V$ over $k$, there is a unique isomorphism $R_{l}(V) \simeq \bigoplus_{i} H_{l}^{i}(V)[-i]$ inducing the 
identity map on cohomology and such that $P^{i}\left(\varpi_{V}\right)$ is the $i^{\text {th }}$ projection map. Here $P^{i}$ is the polynomial in (2). This shows that

$$
R \Gamma(V) \simeq R_{l}(R V)
$$

when $V$ is projective and smooth. For an arbitrary $V$, we choose a simplicial resolution $V \stackrel{f}{\longleftarrow} U_{\bullet} \stackrel{j}{\longrightarrow} X_{\bullet}$ of $V$. Because (10) holds for smooth projective varieties,

$$
R \Gamma\left(U_{\bullet} \stackrel{j}{\longrightarrow} X_{\bullet}\right) \simeq R_{l}\left(R\left(U_{\bullet} \stackrel{j}{\longrightarrow} X_{\bullet}\right)\right)
$$

Moreover,

$$
\begin{aligned}
R\left(U_{\bullet} \stackrel{j}{\longrightarrow} X_{\bullet}\right) & \simeq R(V) \quad(\text { definition of } R(V)) \\
R \Gamma\left(U_{\bullet} \stackrel{j}{\longrightarrow} X_{\bullet}\right) & \simeq R \Gamma(V) \quad(\text { Saint-Donat 1973, 4.3.2; also Huber 1995, 1.1.3), }
\end{aligned}
$$

and so (10) holds for all varieties.

Statement (d), $l=p$. Let $R$ be the Raynaud ring, and $D(R)$ the derived category of the category of graded $R$-modules (Illusie 1983, 2.1). For a smooth projective variety $X$ over $k$, let $W \Omega_{X}^{\bullet}$ be the de Rham-Witt complex on $X$, and let $R \Gamma\left(W \Omega_{X}^{\bullet}\right)$ be its image under the derived functor of $\Gamma=\Gamma(X,-)$. Then $R \Gamma\left(W \Omega_{X}^{\bullet}\right)$ lies in the full subcategory $D_{c}^{b}(R)$ of $D(R)$ consisting of bounded $R$ complexes whose cohomology modules are coherent (Illusie and Raynaud 1983, II $2.2)$, and $H^{i}\left(R \Gamma\left(W \Omega_{X}^{\bullet}\right) \simeq H_{\text {crys }}^{i}(X / W)\right.$. When we endow $D_{c}^{b}(R)$ with Ekedahl's $t$-structure (Illusie 1983, 2.4.8) and quotient out by torsion objects, we obtain a $\mathbb{Q}_{p}$-linear $t$-category $D_{c}^{b}(R)_{\mathbb{Q}}$ whose heart is the category $\mathcal{R}\left(k, \mathbb{Q}_{p}\right)$ of $F$-isocrystals over $k$. It is known that $D_{c}^{b}(R)_{\mathbb{Q}} \simeq D_{\mathcal{R}}^{b}\left(B_{\sigma}[F]\right)$ (derived category of bounded complexes of $B_{\sigma}[F]$-modules whose cohomology groups are $F$-isocrystals over $k$; recall $B=W \otimes \mathbb{Q}$ and that $B_{\sigma}[F]$ is the twisted polynomial ring). Define

$$
R_{p}: D^{b}\left(\mathcal{M}_{\text {num }}(k)\right) \rightarrow D^{b}\left(\mathcal{R}\left(k, \mathbb{Q}_{p}\right)\right) \rightarrow D_{\mathcal{R}}^{b}\left(B_{\sigma}[F]\right) \simeq D_{c}^{b}(R)_{\mathbb{Q}}
$$

to be the composite of the derived functor of the fibre functor $\mathcal{M}_{\text {num }}(k) \rightarrow$ $\mathcal{R}\left(k, \mathbb{Q}_{p}\right)$ with the natural functors. The proof can now be completed as in the case $l \neq p$ except that the reference to Saint-Donat 1973 must be replaced by a reference to Tsuzuki 2003. 
REMARK 5.4 Statement (c) and (d) of the theorem are very strong. Consider, for example, a closed subvariety $Z$ of codimension $r$ in a smooth projective variety $X$ and a smooth alteration $\tilde{Z} \rightarrow Z$. Then the theorem says that there is an exact sequence

$$
h^{i-2 r}(\tilde{Z})(r) \rightarrow h^{i}(X) \rightarrow h^{i}(U), \quad U=X \backslash Z,
$$

whose $l$-adic realization is the sequence in (1.14) for $l \neq p$.

\section{Application.}

5.5 Using (c) and (d), we can extend the definition of $\mathbb{Q}_{p}$ cohomology (Milne 1986a, p309) from smooth projective varieties to all varieties, namely, for any variety $X$ over $k$, define

$$
H^{i}\left(X, \mathbb{Q}_{p}(r)\right)=\operatorname{Hom}_{\mathcal{D}\left(k ; \mathbb{Q}_{p}\right)}\left(\mathbb{1}, R_{p}(R X)(r)[i]\right) .
$$

The main theorem of Milne and Ramachandran 2005 shows that this agrees with the original definition when $X$ is smooth and projective.

\section{The $\mathbb{Q}$-algebra of correspondences at the generic point}

In this section, $k=\mathbb{F}_{q}$ and we assume that the Tate conjecture holds over $k$ and that numerical equivalence equals rational equivalence $(\mathbb{Q}$-coefficients). We allow $l=p$.

\section{Effective motives}

Let $\mathcal{M}^{\text {eff }}(k)$ be the category of effective motives given by Grothendieck's construction using algebraic classes modulo numerical equivalence as correspondences. It is an abelian nonrigid tensor category, and we let $\mathcal{D}^{\text {eff }}(k)=D^{b}\left(\mathcal{M}^{\text {eff }}(k)\right)$. Much of Theorem 5.3 continues to hold. In particular, attached to a smooth projective variety $X$ and an open subvariety $U$, there is a well-defined restriction map 
$h^{i}(X) \rightarrow h^{i}(U)$ whose $l$-adic realization is $H_{l}^{i}(X) \rightarrow H_{l}^{i}(U)$ (cf. 5.4, 5.5). We define

$$
F_{a}^{r} h^{i}(X)=\bigcup_{U} \operatorname{Ker}\left(h^{i}(X) \rightarrow h^{i}(U)\right)
$$

where $U$ runs over the open subvarieties of $X$ such that $X \backslash U$ is of codimension at least $r$.

Proposition 6.1 For all $l$ (including $l=p$ )

$$
R_{l}\left(F_{a}^{r} h^{i}(X)\right)=F_{b}^{r} H_{l}^{i}(X) .
$$

Proof. The functor $R_{l}$ is exact, and so

$$
R_{l}\left(F_{a}^{r} h^{i}(X)\right)=F_{a}^{r} H_{l}^{i}(X)
$$

Therefore, the statement follows from the generalized Tate conjecture $(1.10,1.14$, $1.16)$.

Corollary 6.2 For any smooth projective variety $X$ over $k, F_{a}^{r} h^{i}(X)$ is the largest effective submotive of $h^{i}(X)$ of the form $M(-r)$ for some effective motive $M$.

Proof. Obvious.

\section{Definition of the $\mathbb{Q}$-algebra of correspondences at the generic point}

In this subsection, we translate some definitions and results of Beilinson 2002 into our context. Let $X$ be a connected algebraic variety of dimension $n$ over a finite field $k$, and let $\eta$ be its generic point. Define

$$
C H^{n}(\eta \times \eta)=\lim _{\longrightarrow} C H^{n}(U \times U),
$$

where $U$ runs over the open subvarieties of $X$. Following Beilinson 2002, 1.4, we define

$$
A(X)=C H^{n}(\eta \times \eta) \otimes \mathbb{Q} .
$$

Composition of correspondences makes $A(X)$ into an associative $\mathbb{Q}$-algebra, called the $\mathbb{Q}$-algebra of correspondences at the generic point. 
Denote by $\bar{h}^{n}(X)$ the image of the canonical map $h^{n}(X) \rightarrow h^{n}(\eta)$ (ind object of $\left.\mathcal{M}^{\mathrm{eff}}(k)\right)$.

TheOREM 6.3 For any connected smooth projective varieties $X, X^{\prime}$ of dimension $n$ over $k$, the map

$$
C H^{n}\left(\eta^{\prime} \times \eta\right) \otimes \mathbb{Q} \rightarrow \operatorname{Hom}\left(\bar{h}^{n}(\eta), \bar{h}^{n}\left(\eta^{\prime}\right)\right)
$$

is an isomorphism.

Proof. Beilinson's proof $(2002,4.9)$ applies in our context.

Corollary 6.4 For any connected smooth projective variety $X$ of dimension $n$ over $k$, there is a canonical isomorphism of $\mathbb{Q}$-algebras

$$
A(X) \simeq \operatorname{End}\left(\bar{h}^{n}(X)\right) \text {. }
$$

Proof. It is only necessary to observe that composition of correspondences corresponds to composition of endomorphisms (Beilinson 2002, 4.10).

Corollary 6.5 The $\mathbb{Q}$-algebra $A(X)$ is finite-dimensional and semisimple.

Proof. Immediate from (6.4) because $\mathcal{M}^{\text {eff }}(k)$ is a semisimple category over $\mathbb{Q}$ with finite-dimensional Homs.

\section{Calculation of the $\mathbb{Q}$-algebra of correspondences at the generic point}

Proposition 6.6 For a connected curve $X$ over $k$,

$$
A(X) \simeq \operatorname{End}(J) \otimes \mathbb{Q}
$$

where $J$ is the Jacobian of a smooth complete model of $X$.

Proof. As $X$ is geometrically reduced, its smooth locus $X^{\prime}$ can be embedded in a smooth projective curve $Y$, and $X^{\prime} \hookrightarrow Y$ is uniquely determined up to a unique isomorphism. As

$$
A(X) \simeq A\left(X^{\prime}\right) \simeq A(Y)
$$


we may as well assume that $X$ itself is smooth and projective. For any nonempty open $U$, the map $h^{1}(X) \rightarrow h^{1}(U)$ is injective because $H_{l}^{1}(X) \rightarrow H_{l}^{1}(U)$ is injective, and so $\bar{h}^{1}(X)=h^{1}(X)$. Therefore, $A(X) \simeq \operatorname{End}\left(h^{1}(X)\right)$, and it follows from the isomorphism

$$
C H^{1}(X \times X) \simeq C H^{1}(X) \oplus C H^{1}(X) \oplus \operatorname{End}(J)
$$

(Weil 1948a), that

$$
\operatorname{End}\left(h^{1}(X)\right) \simeq \operatorname{End}(J) \otimes \mathbb{Q}
$$

For a connected smooth projective variety $X$ of dimension $n$ over $k$, define ${ }^{8}$

$$
\bar{H}_{l}^{n}(X)=H_{l}^{n}(X) / F_{b}^{0} H_{l}^{n}(X) .
$$

For $l \neq p$, the quotient map $H_{l}^{n}(X) \rightarrow \bar{H}_{l}^{n}(X)$ defines an isomorphism of $\bar{H}_{l}^{n}(X)$ with the Tate substructure of $H_{l}^{n}(X)$ whose Frobenius eigenvalues $\alpha$ are such that $a / q$ is not an algebraic integer. The quotient map $H_{p}^{n}(X) \rightarrow \bar{H}_{p}^{n}(X)$ can be identified with the map

$$
H^{n}\left(X, W \Omega^{\bullet}\right)_{\mathbb{Q}} \rightarrow H^{n}\left(X, W \mathcal{O}_{X}\right)_{\mathbb{Q}} \simeq H_{p}^{n}(X)_{[0,1[}
$$

(Illusie 1979, II 3.5.3, p616).

Proposition 6.7 For all primes $l$ (including $l=p$ ),

$$
R_{l}\left(\bar{h}^{n}(X)\right) \simeq \bar{H}_{l}^{n}(X)
$$

Proof. Clearly,

$$
0 \rightarrow F_{a}^{0} h^{n}(X) \rightarrow h^{n}(X) \rightarrow \bar{h}^{n}(X) \rightarrow 0
$$

is exact. On applying the exact functor $R_{l}$, this gives an exact sequence

$$
0 \rightarrow F_{b}^{0} H_{l}^{n}(X) \rightarrow H_{l}^{n}(X) \rightarrow \bar{H}_{l}^{n}(X) \rightarrow 0
$$

by $(6.4)$.

\footnotetext{
${ }^{8}$ For $l \neq p$, this is $G r^{0} H_{l}^{n}(X)$, the "composante pure de niveau $n$ " of $H_{l}^{n}(X)$, of Grothendieck (1968, p162).
} 
Theorem 6.8 For all primes $l$ (including $l=p$ ),

$$
A(X) \otimes \mathbb{Q}_{l} \simeq \operatorname{End}\left(\bar{H}_{l}^{n}(X)\right)
$$

(endomorphisms of $\bar{H}_{l}^{n}(X)$ as a Tate structure when $l \neq p$; endomorphisms of $\bar{H}_{p}^{n}(X)$ as an $F$-isocrystal when $l=p$ ).

Proof. Follows from Proposition 6.7 and the fact that $R_{l}$ defines isomorphisms

$$
\operatorname{Hom}(M, N) \otimes_{\mathbb{Q}} \mathbb{Q}_{l} \simeq \operatorname{Hom}\left(R_{l} M, R_{l} N\right) .
$$

ExAmple 6.9 If $H^{n}\left(X, W \mathcal{O}_{X}\right)$ is torsion, then $A(X)=0$. This is the case, for example, if $X$ is a supersingular abelian surface, a supersingular $K 3$ surface, or an Enriques surface (Illusie 1979, 7.1, 7.2, 7.3).

REMARK 6.10 It is possible to recover the rank of a motive $M$ from its endomorphism algebra $\operatorname{End}(M)$. According to the Wedderburn theorems,

$$
\operatorname{End}(M)=\prod_{j} M_{r_{j}}\left(D_{j}\right)
$$

with each $D_{j}$ a division algebra over $\mathbb{Q}$. If $Z_{j}$ is the centre of $D_{j}$, then

$$
\operatorname{rank}(M)=\sum_{j} r_{j} \cdot\left[Z_{j}: \mathbb{Q}\right] \cdot\left[D_{j}: Z_{j}\right]^{1 / 2}
$$

REMARK 6.11 Since $A(X)$ is a birational invariant, (6.4) and (6.10) show that the rank of $\bar{h}^{n}(X)(n=\operatorname{dim} X)$ is a birational invariant of connected smooth projective varieties. Hence the same is true of its $p$-adic realization, i.e.,

$$
\operatorname{rank} H^{n}\left(X, W \mathcal{O}_{X}\right)=\operatorname{rank} H^{0}\left(X, W \Omega_{X}^{n}\right)
$$

is a birational invariant of connected smooth projective varieties over a finite field. Of course, it is classical that

$$
\operatorname{dim} H^{n}\left(X, \mathcal{O}_{X}\right)=\operatorname{dim} H^{0}\left(X, \Omega_{X}^{n}\right)
$$

is a birational invariant (Hartshorne 1977, II Ex 8.8), but

$$
\operatorname{rank} H^{n}\left(X, W \mathcal{O}_{X}\right) \neq \operatorname{dim} H^{n}\left(X, \mathcal{O}_{X}\right),
$$


for example, when $n=2$ and $X$ is a supersingular abelian surface. Illusie (1979, II 2.18, p614) proves that $H^{0}\left(X, W \Omega_{X}^{n}\right)$ is of finite-type over $W$ with $F$ acting as an automorphism. The formal $p$-divisible group $G$ with Cartier module $H^{0}\left(X, W \Omega_{X}^{n}\right) /$ torsion has

$$
\operatorname{dim}(G)=\operatorname{rank}(G)=\operatorname{rank} H^{0}\left(X, W \Omega_{X}^{n}\right)
$$

(cf. ibid. II 4.4, p621) and so $\operatorname{dim}(G)$ and $\operatorname{rank}(G)$ are also birational invariants.

\section{Explicit description of $A(X)$}

6.12 Let $X$ be a smooth projective variety over $k$, and let $\left(\alpha_{i}\right)_{1 \leq i \leq \beta_{n}}$ be the family of eigenvalues of $\varpi_{X}$ on $H_{l}^{n}(X)$. Then the family $S(X)$ of eigenvalues of $\varpi_{\bar{h}^{n}(X)}$ consists of the $\alpha_{i}$ for which $\alpha_{i} / q$ is not an algebraic integer. Therefore, by Milne 1994, 2.14-2.15, the semisimple $\mathbb{Q}$-algebra $A(X)=\operatorname{End}(\bar{h}(X))$ has the following description. Let $o_{1}, \ldots, o_{s}$ be the distinct orbits for the action of $\operatorname{Gal}\left(\mathbb{Q}^{\text {al }} / \mathbb{Q}\right)$ on $S(X)$ and let $r_{j}$ be the multiplicity of $o_{j}$ :

$$
F(X)=\coprod_{j} r_{j} o_{j}
$$

Then

$$
\bar{h}^{n}(X)=\sum_{j} r_{j} N_{j}
$$

where $N_{j}$ is a simple motive with Frobenius eigenvalues the elements of $o_{j}$, and

$$
A(X) \simeq \prod_{j} M_{r_{j}}\left(\operatorname{End}\left(N_{j}\right)\right) .
$$

Let $\alpha \in o_{j}$. Then $\operatorname{End}\left(N_{j}\right)$ is isomorphic to a central simple algebra $D_{j}$ over $Z_{j}=\mathbb{Q}[\alpha]$ with invariants (at the primes $v$ of $\mathbb{Q}[\alpha]$ )

$$
\operatorname{inv}_{v}\left(D_{j}\right)= \begin{cases}\frac{1}{2} & \text { if } v \text { is real and } n \text { is odd } \\ \frac{\operatorname{ord}_{v}(\alpha)}{\operatorname{ord}_{v}(q)} \cdot\left[\mathbb{Q}[\alpha]_{v}: \mathbb{Q}_{p}\right] & \text { if } v \mid p \\ 0 & \text { otherwise. }\end{cases}
$$

Therefore, the degree $[\mathbb{Q}[\alpha]: \mathbb{Q}]$ is the order of $o_{i}$, and the degree $\left[D_{j}: \mathbb{Q}[\alpha]\right]=$ $e^{2}$ where $e$ is the least common denominator of the numbers $\operatorname{inv}_{v}\left(D_{j}\right)$.

Following Beilinson (2002, p37), the pessimists will be tempted to look for counter-examples to the above calculations in order to ruin the conjectures. 


\section{$7 \quad$ Base fields algebraic over a finite field}

Let $k$ be a subfield of $\mathbb{F}$, and assume that the Tate conjecture holds and numerical equivalence equals rational equivalence ( $\mathbb{Q}$-coefficients) for finite subfields of $k$. When we define the various categories for $k$ to be the 2-category direct limits of the categories for $k^{\prime}$ with $k^{\prime}$ running over the finite subfields of $k$, then these categories for $k$ inherit the properties of the corresponding categories for $k^{\prime}$.

\section{References}

ANDRÉ, Y. 2004. Une introduction aux motifs (motifs purs, motifs mixtes, périodes), volume 17 of Panoramas et Synthèses. Société Mathématique de France, Paris.

Beilinson, A. 2002. Remarks on $n$-motives and correspondences at the generic point, pp. 3546. In Motives, polylogarithms and Hodge theory, Part I (Irvine, CA, 1998), volume 3 of Int. Press Lect. Ser. Int. Press, Somerville, MA.

Beilinson, A. A. 1987. On the derived category of perverse sheaves, pp. 27-41. In Ktheory, arithmetic and geometry (Moscow, 1984-1986), volume 1289 of Lecture Notes in Math. Springer, Berlin.

Beilinson, A. A., Bernstein, J., And Deligne, P. 1982. Faisceaux pervers, pp. 5-171. In Analysis and topology on singular spaces, I (Luminy, 1981), volume 100 of Astérisque. Soc. Math. France, Paris. Cited as BBD.

Berthelot, P. 1997. Altérations de variétés algébriques (d'après A. J. de Jong). Astérisque pp. Exp. No. 815, 5, 273-311.

DE Jong, A. J. 1996. Smoothness, semi-stability and alterations. Inst. Hautes Études Sci. Publ. Math. pp. 51-93.

Deligne, P. 1968. Théorème de Lefschetz et critères de dégénérescence de suites spectrales. Inst. Hautes Études Sci. Publ. Math. pp. 259-278.

Deligne, P. 1974a. La conjecture de Weil. I. Inst. Hautes Études Sci. Publ. Math. pp. 273-307.

Deligne, P. 1974b. Théorie de Hodge. III. Inst. Hautes Études Sci. Publ. Math. pp. 5-77.

Deligne, P. 1980. La conjecture de Weil. II. Inst. Hautes Études Sci. Publ. Math. pp. 137-252.

Deligne, P. 1989. Le groupe fondamental de la droite projective moins trois points, pp. 79 297. In Galois groups over mathbfQ (Berkeley, CA, 1987), volume 16 of Math. Sci. Res. Inst. Publ. Springer, New York. 
Deligne, P. 1990. Catégories tannakiennes, pp. 111-195. In The Grothendieck Festschrift, Vol. II, Progr. Math. Birkhäuser Boston, Boston, MA.

Deligne, P. 1994. A quoi servent les motifs?, pp. 143-161. In Motives (Seattle, WA, 1991), volume 55 of Proc. Sympos. Pure Math. Amer. Math. Soc., Providence, RI.

Deligne, P. And Milne, J. S. 1982. Tannakian categories, pp. 101-228. In Hodge cycles, motives, and Shimura varieties, Lecture Notes in Mathematics 900. Springer-Verlag, Berlin.

Fontaine, J.-M. And Mazur, B. 1995. Geometric Galois representations, pp. 41-78. In Elliptic curves, modular forms, \& Fermat's last theorem (Hong Kong, 1993), Ser. Number Theory, I. Internat. Press, Cambridge, MA.

GabBer, O. 2001. On space filling curves and Albanese varieties. Geom. Funct. Anal. 11:11921200.

Geisser, T. 1998. Tate's conjecture, algebraic cycles and rational $K$-theory in characteristic $p$. K-Theory 13:109-122.

Gelfand, S. I. And Manin, Y. I. 1996. Methods of homological algebra. Springer-Verlag, Berlin.

Grothendieck, A. 1968. Le groupe de Brauer. III. Exemples et compléments, pp. 88-188. In Dix Exposés sur la Cohomologie des Schémas. North-Holland, Amsterdam. Available at www.grothendieck-circle.org.

Grothendieck, A. 1969. Hodge's general conjecture is false for trivial reasons. Topology 8:299-303. Available at www.grothendieck-circle.org.

Hanamura, M. 1995. Mixed motives and algebraic cycles. I. Math. Res. Lett. 2:811-821.

Hanamura, M. 1999. Mixed motives and algebraic cycles. III. Math. Res. Lett. 6:61-82.

Hanamura, M. 2004. Mixed motives and algebraic cycles. II. Invent. Math. 158:105-179.

Hartshorne, R. 1977. Algebraic geometry. Springer-Verlag, New York.

Hiller, H. L. 1981. A-rings and algebraic K-theory. J. Pure Appl. Algebra 20:241-266.

HondA, T. 1968. Isogeny classes of abelian varieties over finite fields. J. Math. Soc. Japan 20:83-95.

Huber, A. 1995. Mixed motives and their realization in derived categories, volume 1604 of Lecture Notes in Mathematics. Springer-Verlag, Berlin.

Illusie, L. 1979. Complexe de de Rham-Witt et cohomologie cristalline. Ann. Sci. École Norm. Sup. (4) 12:501-661. 
ILlusie, L. 1983. Finiteness, duality, and Künneth theorems in the cohomology of the de Rham-Witt complex, pp. 20-72. In Algebraic geometry (Tokyo/Kyoto, 1982), volume 1016 of Lecture Notes in Math. Springer, Berlin.

Illusie, L. And Raynaud, M. 1983. Les suites spectrales associées au complexe de de RhamWitt. Inst. Hautes Études Sci. Publ. Math. pp. 73-212.

Jannsen, U. 1992. Motives, numerical equivalence, and semi-simplicity. Invent. Math. 107:447452.

JAnNSEN, U. 2000. Equivalence relations on algebraic cycles, pp. 225-260. In The arithmetic and geometry of algebraic cycles (Banff, AB, 1998), volume 548 of NATO Sci. Ser. C Math. Phys. Sci. Kluwer Acad. Publ., Dordrecht.

KAHN, B. 2002. Number of points of function fields over finite fields. arxiv.nt/0210202.

Langlands, R. P. and Rapoport, M. 1987. Shimuravarietäten und Gerben. J. Reine Angew. Math. 378:113-220. Available online at the Langlands Archive.

Levine, M. 1998. Mixed motives, volume 57 of Mathematical Surveys and Monographs. American Mathematical Society, Providence, RI.

Mazza, C., Voevodsky, V., And Weibel, C. 2006. Lecture notes on motivic cohomology, volume 2 of Clay Mathematics Monographs. American Mathematical Society, Providence, RI.

Milne, J. S. 1980. Etale cohomology, volume 33 of Princeton Mathematical Series. Princeton University Press, Princeton, N.J.

Milne, J. S. 1986a. Arithmetic duality theorems, volume 1 of Perspectives in Mathematics. Academic Press Inc., Boston, MA. (Second edition, BookSurge Publishers, 2006).

Milne, J. S. 1986b. Values of zeta functions of varieties over finite fields. Amer. J. Math. 108:297-360.

Milne, J. S. 1994. Motives over finite fields, pp. 401-459. In Motives (Seattle, WA, 1991), Proc. Sympos. Pure Math. Amer. Math. Soc., Providence, RI.

Milne, J. S. 1999. Lefschetz motives and the Tate conjecture. Compositio Math. 117:45-76.

Milne, J. S. 2003. Gerbes and abelian motives. Preprint available at the author's webpage and at arXiv:math.AG/0301304).

Milne, J. S. 2007. Rational Tate classes. Submitted for publication; arXive:0707.3617.

Milne, J. S. And Ramachandran, N. 2005. The de Rham-Witt and $\mathbb{Z}_{p}$-cohomologies of an algebraic variety. Adv. Math 198:36-42. 
Quillen, D. 1973. Higher algebraic K-theory. I, pp. 85-147. Lecture Notes in Math., Vol. 341. In Algebraic $K$-theory, I: Higher $K$-theories (Proc. Conf., Battelle Memorial Inst., Seattle, Wash., 1972). Springer, Berlin.

SaAvedra Rivano, N. 1972. Catégories Tannakiennes. Springer-Verlag, Berlin.

Saint-Donat, B. 1973. Appendice to Exposé XVII, pp. 462-480. In Théorie des topos et cohomologie étale des schémas (SGA 4, 1963/64). Springer-Verlag, Berlin.

Schoen, C. 1989. Cyclic covers of $\mathbf{P}^{v}$ branched along $v+2$ hyperplanes and the generalized Hodge conjecture for certain abelian varieties, pp. 137-154. In Arithmetic of complex manifolds (Erlangen, 1988), volume 1399 of Lecture Notes in Math. Springer, Berlin.

SEILER, W. K. 1988. $\lambda$-rings and Adams operations in algebraic $K$-theory, pp. 93-102. In Berlinson's conjectures on special values of $L$-functions, volume 4 of Perspect. Math. Academic Press, Boston, MA.

Soulé, C. 1984. Groupes de Chow et K-théorie de variétés sur un corps fini. Math. Ann. 268:317-345.

Soulé, C. 1985. Opérations en K-théorie algébrique. Canad. J. Math. 37:488-550.

TAte, J. T. 1965. Algebraic cycles and poles of zeta functions, pp. 93-110. In Arithmetical Algebraic Geometry (Proc. Conf. Purdue Univ., 1963). Harper \& Row, New York.

TATE, J. T. 1968. Classes d'isogénie des variétés abéliennes sur un corps fini (d'après T. Honda). Séminaire Bourbaki: Vol. 1968/69, Expose 352.

TAte, J. T. 1994. Conjectures on algebraic cycles in l-adic cohomology, pp. 71-83. In Motives (Seattle, WA, 1991), volume 55 of Proc. Sympos. Pure Math. Amer. Math. Soc., Providence, RI.

Tsuzuki, N. 2003. Cohomological descent of rigid cohomology for proper coverings. Invent. Math. 151:101-133.

VAn Den Bergh, M. 2004. A remark on a theorem by Deligne. Proc. Amer. Math. Soc. $132: 2857-2858$.

VERDIER, J.-L. 1977. Categories derivees, quelques resultat (etat 0).

Verdier, J.-L. 1996. Des catégories dérivées des catégories abéliennes. Astérisque pp. xii+253 pp. (1997).

Voevodsky, V. 2000. Triangulated categories of motives over a field, pp. 188-238. In Cycles, transfers, and motivic homology theories, volume 143 of Ann. of Math. Stud. Princeton Univ. Press, Princeton, NJ. 
Weil, A. 1948a. Sur les courbes algébriques et les variétés qui s'en déduisent. Actualités Sci. Ind., no. 1041 = Publ. Inst. Math. Univ. Strasbourg 7 (1945). Hermann et Cie., Paris.

WeIL, A. 1948b. Variétés abéliennes et courbes algébriques. Actualités Sci. Ind., no. $1064=$ Publ. Inst. Math. Univ. Strasbourg 8 (1946). Hermann \& Cie., Paris.

James S. Milne

Mathematics Department, University of Michigan

Ann Arbor, MI 48109, USA

E-mail: jmilne@umich.edu

Webpage: www.jmilne.org/math/

Niranjan Ramachandran

Mathematics Department, University of Maryland

College Park, MD 20742, USA

Email: atma@math.umd.edu

Webpage: www.math.umd.edu/ atma/ 